\title{
BMJ Open Protocol for a scoping review on the methods for engaging long-term care residents with dementia in research and guideline development
}

\author{
Caitlin McArthur (D , , ${ }^{1}$ Adria Quigley, ${ }^{2}$ Rebecca Affoo, ${ }^{3}$ Marie Earl, ${ }^{1}$ Elaine Moody ${ }^{4}$
}

To cite: McArthur C, Quigley A, Affoo R, et al. Protocol for a scoping review on the methods for engaging long-term care residents with dementia in research and guideline development. BMJ Open 2021;11:e051602. doi:10.1136/ bmjopen-2021-051602

- Prepublication history and additional supplemental material for this paper are available online. To view these files, please visit the journal online. (http://dx.doi.org/10.1136/ bmjopen-2021-051602)

Received 24 March 2021 Accepted 17 August 2021

Check for updates

(c) Author(s) (or their employer(s)) 2021. Re-use permitted under CC BY-NC. No commercial re-use. See rights and permissions. Published by BMJ.

${ }^{1}$ School of Physiotherapy, Dalhousie University, Halifax, Nova Scotia, Canada ${ }^{2}$ Center for Outcomes Research and Evaluation (CORE), McGill University Health Centre, Montreal, Québec, Canada ${ }^{3}$ School of Communication Sciences and Disorders, Dalhousie University, Halifax, Nova Scotia, Canada

${ }^{4}$ School of Nursing, Dalhousie University, Halifax, Nova Scotia, Canada

Correspondence to Dr Caitlin McArthur; caitlin.mcarthur@dal.ca

\section{ABSTRACT}

Introduction Patient engagement is important when developing health guidelines to ensure high-quality and patient-centred recommendations. However, patient engagement in research and guideline development remains suboptimal, particularly for vulnerable populations, including residents with dementia living in long-term care (LTC) who are often not included in research and guideline development because of perceived and actual challenges with their health, memory, concentration and communication. Optimal strategies and methods for engaging LTC residents with dementia in research and guideline development remain unknown.

Methods and analysis We will conduct a scoping review in accordance with the Preferred Reporting Items for Systematic Reviews and Meta-Analyses (PRISMA) extension to answer the research questions: (1) What methods have been used to engage LTC residents with dementia in research and guideline development? (2) What are the outcomes of resident engagement? (3) What are the barriers and facilitators to resident engagement? Systematic searches for peer-reviewed articles will be conducted in: Academic Search Premier (EBSCO), APA PsycINFO (EBSC0), CINAHL (EBSCO), Medline (OVID), Embase (Elsevier), Web of Science, and Cochrane Database and in grey literature. Two team members will screen articles and extract data. Results will be presented according to the research question they address. We will engage stakeholders including residents, family members, healthcare providers and representatives from relevant organisations throughout the study.

Ethics and dissemination The scoping review will synthesise what is known about resident engagement in research and guideline development. It may identify gaps in the literature about the optimal methods to engage residents in performing research and developing guidelines and reveal opportunities for new methods. The results will be helpful for researchers and policymakers seeking to develop guidelines and researchers engaging in topics that reflect the priorities and experiences of people with dementia. Results of the scoping review will be disseminated via publication in a peer-reviewed journal and conference presentations, and a one-page lay summary will be shared with our engaged stakeholders.
Strengths and limitations of this study

- The scoping review will identify gaps in current methods for engaging residents with dementia in long-term care (LTC) in research and guideline development which will delineate opportunities for developing new methods in the future.

- The scoping review will include both academic and grey literature to support improvement of patient engagement in vulnerable populations, such as those living in LTC.

- Scoping reviews inherently provide breadth and not depth on a topic. We will not be able to describe quantitative outcomes of engaging residents with dementia in research and guideline development through meta-analyses.

- Only studies published in English will be included, limiting the scope of the review to work from English-speaking countries of those who can pay for translation services.

- Given initial testing of search strategies in licensed databases, we expect that there will be few articles that included LTC residents. If we have an insufficient number of articles to synthesise results we will broaden our inclusion criteria to include communitydwelling older adults with dementia and report our results by setting (eg, community and LTC).

\section{INTRODUCTION}

Health guidelines such as those for falls prevention, pressure injury prevention or medication reconciliation contain evidencebased recommendations for health practice, public health or health policy. ${ }^{1}$ Many studies and organisations recognise that successful research and guideline development and implementation require the engagement of multiple stakeholders (eg, patients, clinicians and policy-makers) to improve health outcomes. ${ }^{1-4}$ Particularly, patient engagement is essential for the development of high-quality and relevant health practice guidelines and research. ${ }^{1-5}$ Patient engagement is defined by the Canadian Institutes 
of Health Research as 'an approach that involves meaningful and active collaboration in governance, priority setting, conducting research and knowledge translation'. ${ }^{6}$ Patient engagement acknowledges that patients are experts, empowered and informed consumers in healthcare decisions and essential to the development of more patient-centred and trustworthy guidelines. ${ }^{3}$ Patient engagement is easily accessible for researchers and guideline developers as it requires forethought and planning but has minimal financial implications. ${ }^{2}$ Both the guidelines developed and the guideline development process is different with patient engagement, where the conduct of guideline development, scope, inclusion of patient-relevant topics, outcome selection and planned approaches to recommendation development, implementation and dissemination are guided and informed by patient input. ${ }^{3}$ Indeed, previous work suggests that patient engagement fundamentally changes the way research is conceptualised, conducted and disseminated. ${ }^{5}$

Patient engagement affects key elements of research and guideline development, including identifying priority health areas, determining if effects of treatments are meaningful, weighing risks and benefits of treatment options, analysing the impact of costs, and assessing acceptability and feasibility. ${ }^{3}$ Patient engagement influences the inclusion of patient-relevant topics, assessment outcomes selection and approaches to recommendation development, ${ }^{3}$ and helps to establish supportive institutional policies and adherence to principles of respect, trust, reciprocity and colearning. ${ }^{5}$ Despite its importance, patient involvement in research and guideline development remains suboptimal, ${ }^{3}$ particularly for guidelines supporting vulnerable populations, such as those living in long-term care (LTC).

Residents in LTC with dementia are often not included in research and guideline development because of perceived and actual challenges with their health, memory and thinking. Currently, $7.1 \%$ of older adults in Canada live in LTC and it has been estimated that by the year 2036 the proportion of older adults living in institutional care facilities will be more than double. ${ }^{7}$ Residents in LTC live with complex health conditions that affect their physical and cognitive health. ${ }^{8}$ For example, $95 \%$ of residents require assistance with basic activities of daily living such as grooming, dressing, walking, transferring and feeding themselves. From a cognitive perspective, $69 \%$ of residents have a primary diagnosis of dementia ${ }^{8}$ while $87 \%$ experience cognitive impairment as result of other primary diagnoses such as stroke, or traumatic brain injury. ${ }^{9}$ Previous work has identified challenges in engaging LTC residents with dementia in research and guideline development. Residents with dementia were often perceived as incapable of verbally communicating their thoughts and feelings making it difficult to elicit subjective accounts. ${ }^{10}$ Indeed, dementia may lead to vague and empty speech, dwindling vocabulary, impaired linguistic reasoning, changes in word association patterns and disordered discourse impairing residents' abilities to convey meaning to the researchers and guideline developers. ${ }^{10}$

The Canadian Institutes of Health Research defines the term patient in patient engagement as 'an overarching term inclusive of individuals with personal experience with a health issue and informal caregivers, including family and friends'. ${ }^{6}$ In Canada, people living in LTC are referred to as 'residents' and a 'person living with dementia' is the preferred way of talking about a person with dementia. ${ }^{11}$ We do not consider people living with dementia in LTC 'patients', therefore, from here forward we will refer to 'resident engagement' and 'engagement of LTC residents with dementia'.

Despite the identified challenges, involving residents in research and guideline development is essential to ensure the final product is resident centred: the research and guidelines reflect topics and include actions that are important to residents. ${ }^{10}$ However, the best strategies and methods for engaging LTC residents with dementia in research and guideline development remain unknown. Therefore, the overall purpose of this scoping review is to synthesise what is known about methods to engage LTC residents with dementia in health research and guideline development and identify gaps in research where new methods may need to be developed. The specific objectives are to describe the: (1) methods that have been used to engage LTC residents with dementia in research and guideline development; (2) outcomes of engaging LTC residents with dementia in research and guideline development and (3) barriers and facilitators to engaging residents with dementia in research and guideline development. A scoping review methodology was chosen to provide breadth on the topic and to include a variety of publication types including grey literature, as we recognise that organisations may have engaged LTC residents with dementia but may not have published their work in peer-reviewed journals. This scoping review will inform a larger research programme where LTC residents with dementia will be engaged in developing guidelines and guiding the research priorities.

\section{METHODS AND ANALYSIS}

The review will be conducted in accordance with the PRISMA extension for Scoping Reviews (PRISMA-ScR) ${ }^{12}$ (online supplemental appendix 1-PRIMSA-ScR checklist), and the framework and suggestions proposed by Arksey and O'Malley ${ }^{13}$ and Levac et al. ${ }^{14}$

\section{Research questions}

The research team has defined three research questions for the scoping review:

1. What methods have been used to engage LTC residents with dementia in research and guideline development?

2. What are the outcomes of resident engagement?

3. What are the barriers and facilitators to resident engagement? 


\section{Patient and public involvement}

To receive feedback and suggestions on our protocol, we have engaged stakeholders including residents with dementia and family members in LTC, healthcare professionals (therapeutic recreation specialists, physiotherapists, nurses and healthcare aides), and representatives from the Alzheimer's Society of Nova Scotia. We will continue to meet with our stakeholders 3-4 times over the course of the project. Stakeholders provided input on the inclusion and exclusion criteria, research questions and data items to be extracted. Throughout the project, they will continue to provide input on identifying grey literature, interpreting the results, recommendations for resident engagement in future research and guideline development, and mechanisms of disseminating the results.

\section{Information sources \\ Licensed databases}

Systematic searches for peer-reviewed articles will be conducted in the following licensed databases from inception with no language limitations: Academic Search Premier (EBSCO), APA PsycINFO (EBSCO), CINAHL (EBSCO), Medline (OVID), Embase (Elsevier), Web of Science and the Cochrane database. The search strategy will use text and indexing terms to capture the key concepts: resident (patient) engagement, research and guideline development and dementia (see online supplemental file 1) for search filters run in CINAHL). We will test our search strategy with a predetermined relevant article. ${ }^{10}$ Concepts will be combined with the Boolean Operator AND, and the search terms within each concept will be combined with OR. Truncation and phrase symbols will be used when appropriate to capture comprehensive results. The search strategy has been developed and will be executed by a research librarian at Dalhousie University.

\section{Grey literature}

Guided by the Canadian Agency for Drugs and Technologies in Health Grey Matters Tool, ${ }^{15}$ we will search grey literature pertaining to research and guideline development including LTC resident engagement.

\section{Handsearching}

We will handsearch the reference lists of included articles for more relevant citations. We will also identify and contact researchers and opinion leaders in the area of patient engagement for people with dementia to gather additional articles.

\section{Eligibility criteria}

The following study designs will be included: case studies, mixed-methods, prospective, longitudinal, retrospective case-control, randomised controlled trials and quasirandomised clinical or controlled trials. Studies must include LTC residents defined as individuals who reside in a residential home for people who are unable to live independently, requiring access to nursing, personal care, support and/or supervision, with a mean age of 65 years or older and a dementia diagnosis. ${ }^{16}$ However, based on preliminary search results, we expect a very small number of articles where LTC residents with dementia are included in research and guideline development. Thus, if we do not find three or more articles to synthesise, we will extend our inclusion criteria in two ways: (1) to capture engagement of LTC residents with or without dementia, as many residents in LTC have dementia and (2) to capture people with dementia of any age living in the community. Our search strategy does not include LTC as a search term and will, therefore, gather articles including communitydwelling older adults with dementia. Included studies must describe, research or evaluate resident engagement, defined as including residents with dementia in the process of developing healthcare guidelines or research which could include collaborators or partners in planning, execution or dissemination of the guideline or research. ${ }^{15}$ We will exclude articles that are not in English, are editorials, clinical commentaries, lectures, legal cases, letters, newspaper articles, patient education handouts or unpublished literature.

\section{Selection of sources of evidence}

Two team members will review the title and abstract of identified articles and apply the inclusion and exclusion criteria outlined above. We will use the Covidence software to organise and manage all aspects of the review (eg, selection, data extraction). The full texts of articles that meet the inclusion criteria or for which there is inadequate information will be reviewed by the same two team members. Disagreements will be resolved through discussion with a third team member. The screening process will be pilot tested on $5 \%$ of articles, and forms and processes will be modified if there is low agreement (kappa $<0.5$ ) between the two reviewers. We will document why full-text articles are not included, in accordance with the PRIMSA framework. Screening title, abstract and full-text will take approximately 2 months.

\section{Charting the data}

A data extraction form will be designed by the research team, pilot tested on a random sample of 10 articles, and revised as needed. Two team members will use the pretested data extraction form to chart the data from the included articles independently and in duplicate. Disagreement will be resolved by a third team member. Table 1 describes the data that will be extracted from each included study. Charting the data will take approximately 1 month.

\section{Summarising and reporting the findings}

To provide an overall summary of the results, we will use descriptive statistics and infographics, such as bar charts and maps, to visually display year of publication and country of origin. We will then describe the results according to the research question addressed. If we extend the population scope to include people living with 
Table 1 Data items to be extracted

\section{Category}

Summary

\section{Data items to be extracted}
a. Title
b. Author
c. Location (country).
d. Which research question is addressed (1, 2 or 3$)$.
e. Study design.
f. Description of guideline being developed, research being performed.
g. Description of (1) type of dementia of included participants; (2) level of cognitive impairment of included participants (eg, mean MMSE or MoCA score).
h. Description of setting (LTC or community-dwelling should we need to extend our population scope).
i. Mean age of participants.
j. Description of comorbidities, health or functional status of participants (eg, Charlson Comorbidity Index, Frailty Index).
k. Description of care philosophy of home/organisation.

Research question 1: methods of engagement

a. Type of engagement (eg, discussion groups, focus group, survey).

b. Methods to support engagement (eg, methods to support communication, comprehension or expression).

c. Stage of guideline development (based on the 10-step framework for engaging patients in guideline development. ${ }^{18}$

d. Amount of time spent engaged (eg, 1-hour meetings every month).

e. How many residents engaged, percentage of residents within a home involved.

f. Other stakeholders engaged (eg, family members, healthcare providers) and how they were engaged.

g. Recruitment methods for residents engaged (eg, convenience sampling, resident advocates).

$\begin{aligned} & \text { Research question 2: outcomes of } \\ & \text { resident engagement }\end{aligned}$
$\begin{aligned} & \text { b. Resident outcomes (eg, level of engagement, satisfaction with engagement). } \\ & \text { c. LTC staff outcomes (eg, decreased workload). } \\ & \text { d. Research or guideline development outcomes (eg, considered alternative modes of } \\ & \text { delivery, outcomes to assess, domains of information to include, acceptability). } \\ & \text { e. Outcome measures used. }\end{aligned}$

Research question 3: barriers and facilitators to resident engagement

a. Resident level (eg, fluctuating health conditions).

b. Research team level (eg, negative perceptions).

c. Organisational level (eg, within the LTC home).

LTC, long-term care; MMSE, mini-mental state exam; MoCA, montreal congnitive assessment.

dementia in the community (as described above), we will also describe results by the dwelling location (ie, LTC or community dwelling).

Research question 1: methods of engagement

We will provide an overall outline of the methods of engagement in table format where we will describe the type, timing and amount of time of the engagement, how many residents were engaged, other stakeholders who were engaged and how residents were recruited. Descriptions will include the frequency of similar types and timing of engagement, methods of recruitment and other stakeholders engaged. We will also report the range, mean and $\mathrm{SD}$ of amount of time of engagement and how many residents were engaged in the narrative of the results section.

Research question 2: outcomes of engagement

We will describe the engagement outcomes through a narrative report at three different levels: resident, research team and research or guideline development. For example, if residents are more satisfied with a healthcare guideline this will be reported as 'improved satisfaction with guideline' at the resident level. We will identify the outcome measure and report the outcomes of engagement for each included article in a table grouped by level (ie, resident, research team and research or guideline development). In the narrative results section, we will further group the description by articles that report outcomes at the same level using the same outcome measure.

Research question 3: barriers to engagement

Guided by the principles of thematic content analysis, ${ }^{17}$ we will group barriers to engaging residents in research or guideline development into themes and report them by the level at which the barriers exist. A priori, we have identified three levels (ie, resident, research team and 
organisational level). However, should additional levels arise throughout our analysis we will iteratively add them to the description. Summarising and reporting the findings will take approximately 2 months.

\section{Ethics and dissemination}

The scoping review will synthesise what is known about resident engagement in research and guideline development. The review may identify gaps in the literature about the optimal ways to engage residents in research and developing guidelines, identifying opportunities for new methods to be developed. The results of the review will be helpful for researchers and policymakers to guide how residents could be engaged, methods can be adapted or where new methods need to be developed to ensure residents are engaged in research and guideline development in the future.

The results of the scoping review will be disseminated via publication in a peer-reviewed journal and presented at conferences relevant to LTC, patient engagement and the ageing population. We will also prepare a one-page lay summary to be shared with our engaged stakeholders and local LTC homes. Research ethics board approval will not be required for the scoping review or stakeholder involvement in our review.

Acknowledgements The authors would like to acknowledge the contributions of the research librarians, Shelley McKibbon and interns, Allison Patton, and Vinson $\mathrm{Li}$, for assistance in developing the search strategy, and the research assistant, Niousha Alizadeh Saravi, at Dalhousie University.

Contributors CM led development and writing of the protocol with critical input and revisions from $A Q, R A, M E$ and $E M$.

Funding This research was support by a Research Establishment Grant from the Faculty of Health at Dalhousie University (grant number N/A).

Competing interests None declared.

Patient consent for publication Not required.

Provenance and peer review Not commissioned; externally peer reviewed.

Supplemental material This content has been supplied by the author(s). It has not been vetted by BMJ Publishing Group Limited (BMJ) and may not have been peer-reviewed. Any opinions or recommendations discussed are solely those of the author(s) and are not endorsed by BMJ. BMJ disclaims all liability and responsibility arising from any reliance placed on the content. Where the content includes any translated material, BMJ does not warrant the accuracy and reliability of the translations (including but not limited to local regulations, clinical guidelines, terminology, drug names and drug dosages), and is not responsible for any error and/or omissions arising from translation and adaptation or otherwise.

Open access This is an open access article distributed in accordance with the Creative Commons Attribution Non Commercial (CC BY-NC 4.0) license, which permits others to distribute, remix, adapt, build upon this work non-commercially, and license their derivative works on different terms, provided the original work is properly cited, appropriate credit is given, any changes made indicated, and the use is non-commercial. See: http://creativecommons.org/licenses/by-nc/4.0/.

ORCID iD

Caitlin McArthur http://orcid.org/0000-0001-9985-2796

\section{REFERENCES}

1 Petkovic J, Riddle A, Akl EA, et al. Protocol for the development of guidance for stakeholder engagement in health and healthcare Guideline development and implementation. Syst Rev 2020;9:21.

2 Armstrong MJ, Mullins CD, Gronseth GS, et al. Recommendations for patient engagement in guideline development panels: a qualitative focus group study of guideline-naïve patients. PLoS One 2017;12:e0174329.

3 Armstrong MJ, Mullins CD, Gronseth GS, et al. Impact of patient involvement on clinical practice Guideline development: a parallel group study. Implement Sci 2018;13:55.

4 Morin SN, Djekic-Ivankovic M, Funnell L, et al. Patient engagement in clinical guidelines development: input from >1000 members of the Canadian osteoporosis patient network. Osteoporos Int 2020;31:867-74.

5 Kirwan JR, de Wit M, Frank L, et al. Emerging guidelines for patient engagement in research. Value Health 2017;20:481-6.

6 Canadian Institutes of Health Research. Patient engagement in research resource, 2020. Available: https://cihr-irsc.gc.ca/e/51916. html

7 Canadian Institute of Health Information. CCRS quick STATs 2014/2015, 2015. Available: https://www.cihi.ca/en/quick-stats

8 Hirdes JP, Mitchell L, Maxwell CJ, et al. Beyond the 'iron lungs of gerontology': using evidence to shape the future of nursing homes in Canada. Can J Aging 2011;30:371-90.

9 Edvardsson D, Sjögren K, Lood Q, et al. A person-centred and thriving-promoting intervention in nursing homes - study protocol for the U-Age nursing home multi-centre, non-equivalent controlled group before-after trial. BMC Geriatr 2017;17:22.

10 Hubbard G, Downs MG, Tester S. Including older people with dementia in research: challenges and strategies. Aging Ment Health 2003;7:351-62.

11 Alzheimer's Society. Postive language: an alzheimer's soceity guide to talking about dementia, 2018. Available: https:// www.alzheimers.org.uk/sites/default/files/2018-09/Positive\% 20language\%20guide_0.pdf

12 Tricco AC, Lillie E, Zarin W, et al. PRISMA extension for scoping reviews (PRISMA-ScR): checklist and explanation. Ann Intern Med 2018;169:467-73.

13 Arksey H, O'Malley L. Scoping studies: towards a methodological framework. Int J Soc Res Methodol 2005;8:19-32.

14 Levac D, Colquhoun H, O'Brien KK. Scoping studies: advancing the methodology. Implement Sci 2010;5:69.

15 CADTH. Grey matters: a practical tool for searching health-related grey literature, 2021. Available: https://www.cadth.ca/resources/ finding-evidence/grey-matters

16 Canada H. Long-term facilities-based care, 2004. Available: http:// www.hc-sc.gc.ca/hcs-sss/home-domicile/longdur/index-eng.php

17 Graneheim UH, Lundman B. Qualitative content analysis in nursing research: concepts, procedures and measures to achieve trustworthiness. Nurse Educ Today 2004;24:105-12.

18 Armstrong MJ, Rueda J-D, Gronseth GS, et al. Framework for enhancing clinical practice guidelines through continuous patient engagement. Health Expect 2017;20:3-10. 